\title{
Incomplete copolymer degradation of in situ chemotherapy
}

Pierre Bourdillon, MD, MSc ${ }^{1,2,3,4}$, Tanguy Boissenot, PharmD, $\mathrm{PhD}^{5,6}$, Lauriane Goldwirt, PharmD, $\mathrm{PhD}^{7}$, Julien Nicolas, $\mathrm{PhD}^{5,6}$, Caroline Apra, MD, MSc ${ }^{2,4}$, Alexandre Carpentier, $\mathrm{MD}, \mathrm{PhD}^{2,4,8}$

1. Hospices Civils de Lyon, Hospital for neurology and neurosurgery Pierre Wertheimer, Department of Neurosurgery, Lyon, France

2. Brain and spine institute, INSERM U1127, CNRS 7225, Paris, France

3. Université de Lyon, Université Claude Bernard, Lyon, France

4. Sorbonne Universités, Université Pierre et Marie Curie, Paris, France

5. Institut Galien Paris-Sud, UMR CNRS 8612, Faculté de Pharmacie, Paris, France

6. Université Paris-Saclay, Université Paris-sud, Paris, France

7. Assistance publique hôpitaux de Paris, Saint-Louis Hospital, Department of Pharmacology, Paris, France

8. Assistance publique hôpitaux de Paris, Pitié-Salpêtrière Hospital, Department of neurosurgery, Paris, France

\section{Corresponding author:}

Pierre Bourdillon

Department of Neurosurgery, P. Wertheimer hospital, hospices civils de Lyon, 59, Bd Pinel, 69003, Lyon, France

Phone: (+33) 472118900

Fax: (+33) 472357365

pierre.bourdillon@ neurochirurgie.fr

\section{Author Contributions:}

Dr. P Bourdillon: Study concept and design / acquisition of data / analysis and interpretation/ study supervision.

Dr. T Boissenot Acquisition of data.

Dr. L Goldwirt : Revision of the manuscript for important intellectual content. 
Dr. J Nicolas: Acquisition of data/ analysis and interpretation/ critical revision of the manuscript for important intellectual content.

Dr. C Apra: Revision of the manuscript for important intellectual content.

Pr. A Carpentier: Acquisition of data/ critical revision of the manuscript for important intellectual content/ study supervision.

Key-words:

Glioblastoma; carmustine; gliadel; recurrent surgery; BCNU; in situ drug delivery.

\begin{abstract}
:
Introduction: in situ carmustine wafers containing 1,3-bis(2-chloroethyl)-1-nitrosourea (BCNU) are commonly used for the treatment of recurrent glioblastoma to overcome the brainblood barrier. In theory, this chemotherapy diffuses into the adjacent parenchyma and the excipient degrades in maximum 8 weeks but no clinical data confirms this evolution, because patients are rarely operated again.
\end{abstract}

Materials and Methods: a 75-year-old patient was operated twice for recurrent glioblastoma, and a carmustine wafer was implanted during the second surgery. Eleven months later, a third surgery was performed, revealing unexpected incomplete degradation of the wafer. $1 \mathrm{H}-\mathrm{Nuclear}$ Magnetic Resonance was performed to compare this wafer to pure BCNU and to an unused copolymer wafer.

Results: In the used wafer, peaks corresponding to hydrophobic units of the excipient were no longer noticeable, whereas peaks of the hydrophilic units and traces of BCNU were still present. These surprising results could be related to the formation of a hydrophobic membrane around the wafer, thus interfering with the expected diffusion and degradation processes.

Conclusions: The clinical benefit of carmustine wafers in addition to the standard radiochemotherapy remains limited, and in vivo behavior of this treatment is not completely elucidated yet. We found that the wafer may remain after several months. Alternative strategies to deal with the blood-brain barrier, such as drug-loaded liposomes or ultrasound-opening, must be explored to offer larger drug diffusion or allow repetitive delivery. 


\section{Graphical abstract}

\section{Carmustine wafer}

Unexpected non-degraded copolymer
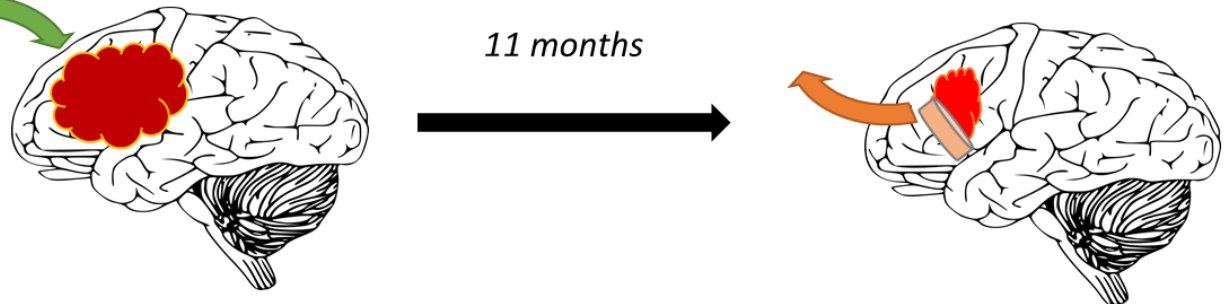


\section{Introduction}

Tight capillary cellular junctions of the blood-brain barrier (BBB) limit drug diffusion from the systemic circulation to the brain parenchyma and prevent the use of numerous chemotherapies for central nervous system neoplasms ${ }^{1}$. Numerous approaches aiming to breaching the BBB, including molecular engineering ${ }^{2,3}$ and mechanical devices ${ }^{4}$, have been developed to answer the challenge of bringing anti-cancer agents ${ }^{5}$ into the brain. In this context, in situ therapies, consisting in direct cell ${ }^{6}$ or $\operatorname{drug}^{7}$ infusion, have also been proposed. Carmustine wafers fall into this last category: they are biodegradable copolymers containing 3.85\% of 1,3-bis(2chloroethyl)-1-nitrosourea (BCNU, also known as carmustine) and are used as an in situ chemotherapy in glioblastoma recurrences and even, more recently, as an adjuvant first-line treatment in newly diagnosed tumors ${ }^{8}$. Each wafer delivers $7.7 \mathrm{mg}$ of carmustine during 5 days with only $1 \%$ of the administered drug remaining in the brain tissue after 7 days ${ }^{9}$. Studies in non-human primates in normal brain without surgical debulking reveal a diffusion of carmustine in a 2 to $10 \mathrm{~mm}$ range around the wafer ${ }^{10}$. The inactive excipient consists in a degradable, random copolymer of 1,3-bis-( $p$-carboxyphenoxy)propane (CPP) and sebacic acid (SA) units in a 20:80 molar ratio connected by anhydride bonds (Figure 1a), termed poly[(1,3bis-(p-carboxyphenoxy)propane)-co-(sebacic acid)], $\mathrm{P}(\mathrm{CPP}-c o-\mathrm{SA}) . \mathrm{P}(\mathrm{CPP}-c o-\mathrm{SA})$ should completely degrade over a period of 6 to 8 weeks ${ }^{9}$. SA contains an aliphatic alkyl chain whereas CPP exhibits 2 phenyl rings per CPP unit. Therefore, as degradation occurs in an aqueous environment, it is expected that bond cleavage will first occur at the SA-SA junctions compared to SA-CPP or CPP-CPP junctions.

Although carmustine wafers have been an important subject of research and discussion, there is no report of in vivo local evolution in a patient, to our knowledge. Here, we report the case of a patient who underwent additional surgery months after carmustine wafers insertion, which allowed us to observe the persistence of the wafer. We provide the Proton Nuclear Magnetic Resonance $\left({ }^{1} \mathrm{H}-\mathrm{NMR}\right)$ analysis of this wafer in order to investigate the possible reasons of its default of resorption.

\section{Materials and Methods}

Patient: A 75-year-old patient was admitted for glioblastoma progression, 11 months after a second resection surgery with carmustine wafer insertion. He had previously received 
fractionated focal irradiation at a dose of 2 Gy per fraction given once daily 5 days per week over a period of 6 weeks, for a total dose of $59.4 \mathrm{~Gy}$ focal radiotherapy and 13 regimens of temozolomide at a dose of $75 \mathrm{mg}$ per square meter per day, given 7 days per week from the first day of radiotherapy until the last day of radiotherapy. After a 4-week break, the patient received 7 cycles of adjuvant chemotherapy according to the standard 5-day schedule every 28 days. The dose was $150 \mathrm{mg}$ per square meter for the first cycle and was increased to $200 \mathrm{mg}$ per square meter beginning with the second cycle (no hematologic toxic effects were noticed). Because he presented local recurrence, his case was discussed in a multidisciplinary neuro-oncological meeting, and he underwent a third surgical resection. During the procedure, we surprisingly found remains of the partially degraded copolymer of the carmustine wafer (Figure 1b). We obtained the patient's written consent to analyze and publish anonymously his data. The wafer's spectrum by ${ }^{1} \mathrm{H}-\mathrm{RMN}$ was compared to BCNU and to an unused copolymer wafer.

Sample treatment: Carmustine wafer extracted from the patient and unused carmustine wafer, Gliadel ${ }^{\circledR}$ (mgi pharma ltd) were dissolved using $1 \mathrm{~mL}$ of solvable ${ }^{\circledR}$ solution (Perkin Elmer). The solution was stirred overnight until complete dissolution. After filtration, solvable ${ }^{\circledR}$ was evaporated and replace by $\mathrm{CDCl}_{3}$ before ${ }^{1} \mathrm{H}-\mathrm{NMR}$ analysis. Carmustine, Bicnu ${ }^{\circledR}$ (emcure pharma UK ltd) was directly dissolved in $\mathrm{CDCl}_{3}$ before ${ }^{1} \mathrm{H}-\mathrm{NMR}$ analysis.

Analytical methods. ${ }^{1} \mathrm{H}-\mathrm{NMR}$ spectroscopy was performed in $5 \mathrm{~mm}$ diameter tubes in $\mathrm{CDCl}_{3}$ at $25{ }^{\circ} \mathrm{C}$ on a Bruker Avance 300 spectrometer at $300 \mathrm{MHz}$. The chemical shift scale was calibrated based on the internal solvent signals $\left(\mathrm{CDCl}_{3}, 7.26 \mathrm{ppm}\right)$.

\section{Results}

The ${ }^{1} \mathrm{H}-\mathrm{RMN}$ analysis of the unused wafer exhibits all expected peaks of CPP (Figure $1 a, b, c$, and $d$ ) and SA (Figure $1 e, f$, and $g$ ) units belonging to the copolymer, and with the right molar ratio between CPP and SA units (Figure 1c). Peaks of BCNU were also detected in the 3.3$4.25 \mathrm{ppm}$ region (Figure 1c). Interestingly, the ${ }^{1} \mathrm{H}-\mathrm{RMN}$ spectrum of the partially degraded wafer showed that peaks corresponding to CPP units (peaks $a-d$ ) were no longer noticeable, whereas peaks $f$ and $g$ belonging to SA units were still present. Note that peak $e$ from SA is not visible, probably because of the poor solvation of the nearby carboxylic acid functions in deuterated chloroform (the solvent used for the NMR study). Traces of BCNU were also detected demonstrating that not all BCNU was released from the water. 
Apart from the unexpected finding of a non-degraded wafer, the incomplete clearance concerning only SA and not CPP units was rather surprising. Given SA is more hydrophilic than CPP, it was supposed to be cleared prior to CPP. Because of their greater hydrophobicity, the CPP-CPP bonds are likely more difficult to be cleaved compared to CPP-SA and SA-SA bonds in an aqueous environment. These results may be related to the potential formation of a hydrophobic membrane around the wafer, which could interfere with the normal degradation process.

\section{Discussion}

The benefit of copolymer wafers in addition to the standard radio-chemotherapy is supported by some controlled studies ${ }^{7}$ but remains limited (inferior to 4 months of progression-free survival). However, the higher postoperative infection rate after copolymer wafers implantation does not affect survival ${ }^{7}$. Although the long-term degradation of carmustine wafers has not been studied in patients, recent imaging studies prospectively investigated the MRI aspect of copolymer wafer during the years following surgery ${ }^{11}$. The kinetics of changes of such images mainly helps to discriminate between a "normal" postoperative evolution and an abscess, a tumor residue or recurrence. A restricted diffusion in MRI reshaping copolymer wafer' outline is known to be noticeable up to one year postoperatively. In light of our report and considering that it is highly uncommon to perform a recurrence glioma surgery distant from the use of copolymer wafer because of the short life expectancy of the patients, we question if such MRI findings could not be related to incomplete degradations of the $\mathrm{P}(\mathrm{CPP}-c o-\mathrm{SA})$. The local consequences of such events are unknown.

\section{Conclusion}

Despite exclusive in situ drug delivery during 5 days between surgery and standard radiochemotherapy, carmustine shows limited efficiency. Our report shows that it could be related to an uncontrolled local tolerance and degradation of the copolymer. This finding underlines the importance of new studies concerning the long-term in vivo degradation of copolymer wafer in human. Moreover, alternative strategies to deal with the blood-brain barrier, such as drugloaded liposomes or opening using ultrasound ${ }^{4}$ must be considered. They could offer larger drug diffusion and repetitive delivery compared to that observed with copolymer wafers ${ }^{9}$. 
Disclosure: the authors declare no conflict of interest.

\section{References}

1. Donelli MG, Zucchetti M, D'Incalci M. Do anticancer agents reach the tumor target in the human brain? Cancer Chemother Pharmacol. 1992;30:251-260.

2. Dove A. Breaching the barrier. Nat Biotechnol. 2008;26:1213-1215.

3. Vastag B. Biotechnology: Crossing the barrier. Nature. 2010;466:916-918.

4. Carpentier A, Canney M, Vignot A, et al. Clinical trial of blood-brain barrier disruption by pulsed ultrasound. Sci Trans1 Med. 2016;8:343re2.

5. Jacus MO, Daryani VM, Harstead KE, Patel YT, Throm SL, Stewart CF. Pharmacokinetic Properties of Anticancer Agents for the Treatment of Central Nervous System Tumors: Update of the Literature. Clin Pharmacokinet. 2016;55:297-311.

6. Gill SS, Patel NK, Hotton GR, et al. Direct brain infusion of glial cell line-derived neurotrophic factor in Parkinson disease. Nat Med. 2003;9:589-595.

7. Pallud J, Audureau E, Noel G, et al. Long-term results of carmustine wafer implantation for newly diagnosed glioblastomas: a controlled propensity-matched analysis of a French multicenter cohort. Neuro Oncol. 2015;17:1609-1619.

8. Qi Z, Xing W, Shao C, Yang C, Wang Z. The role of Gliadel wafers in the treatment of\&amp;nbsp;newly diagnosed GBM: a meta-analysis. Drug Des Devel Ther. 2015;9:3341.

9. Fleming AB, Saltzman WM. Pharmacokinetics of the carmustine implant. Clin Pharmacokinet. 2002;41:403-419.

10. Strasser JF, Fung LK, Eller S, Grossman SA, Saltzman WM. Distribution of 1,3-bis(2chloroethyl)-1-nitrosourea and tracers in the rabbit brain after interstitial delivery by biodegradable polymer implants. J Pharmacol Exp Ther. 1995;275:1647-1655.

11. Ulmer S, Spalek K, Nabavi A, et al. Temporal changes in magnetic resonance imaging characteristics of Gliadel wafers and of the adjacent brain parenchyma. Neuro Oncol. 2012;14:482-490. 

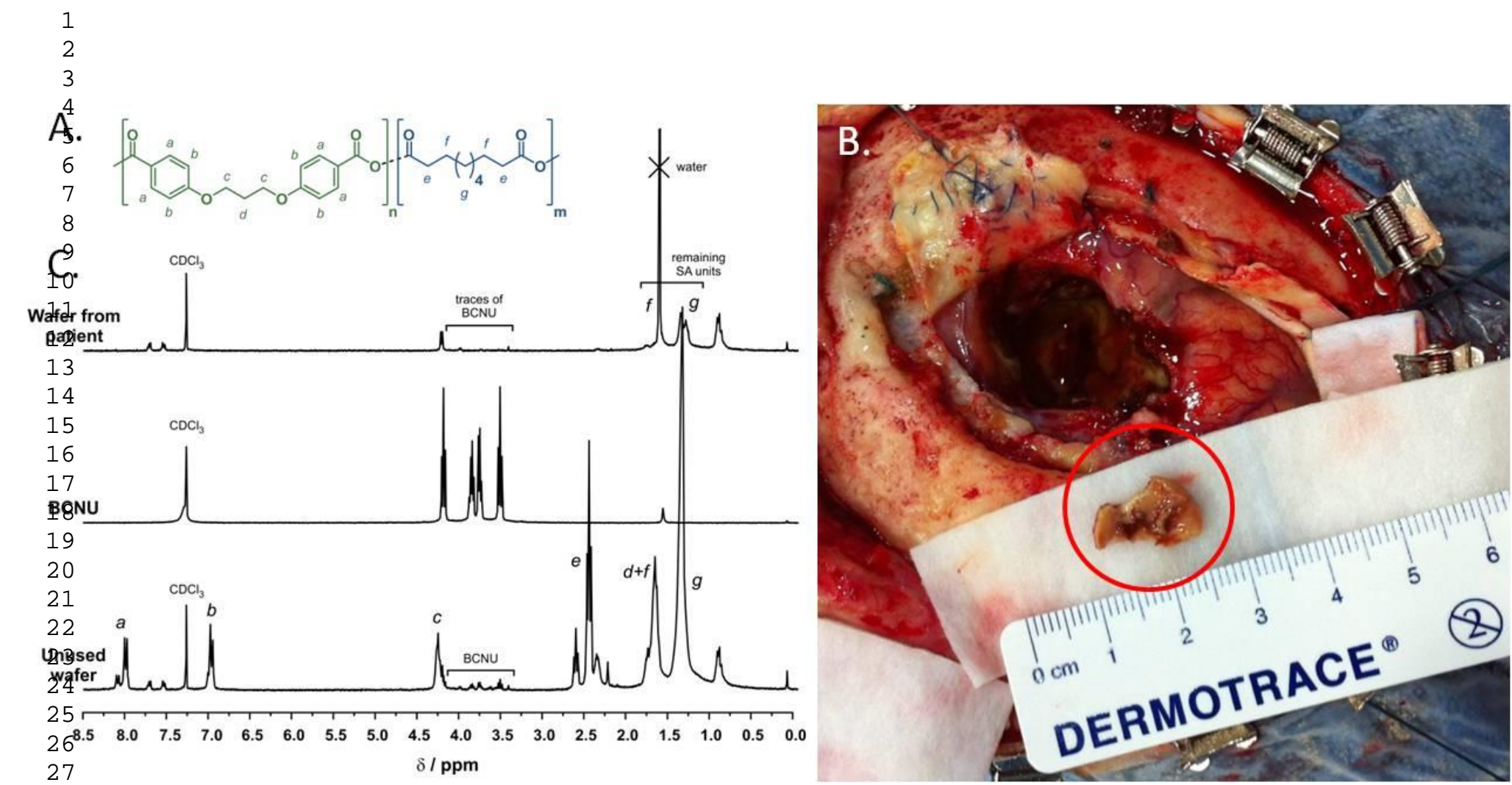

Figure 1: A. Structure of polifeprosan 20: a random copolymer formed by 1,3-bis-( $p$-carboxyphenoxy)propane (CPP, green square) and sebacic acid (SA, blue square) monomers (20:80 molar ratio) connected by anhydride bonds ; B. Circled in red: carmustine wafer one year after a first surgery ; C. 1H RMN analysis of a control carmustine wafer (bottom), BCNU (middle) and the wafer extracted from patient (top). The unused wafer exhibits all expected peaks of CPP (a, b, c, and d) and SA (e, f, and g) units of the copolymer, together with peaks of BCNU in the 3.3-4.25 ppm region (c). The partially degraded wafer shows no peaks corresponding to CPP units, whereas peaks $f$ and $g$ belonging to SA units are still present, with traces of BCNU. Note that peak from SA is not visible, likely because of the poor solvation of the nearby carboxylic acid functions in deuterated chloroform (solvent used for the NMR study). 

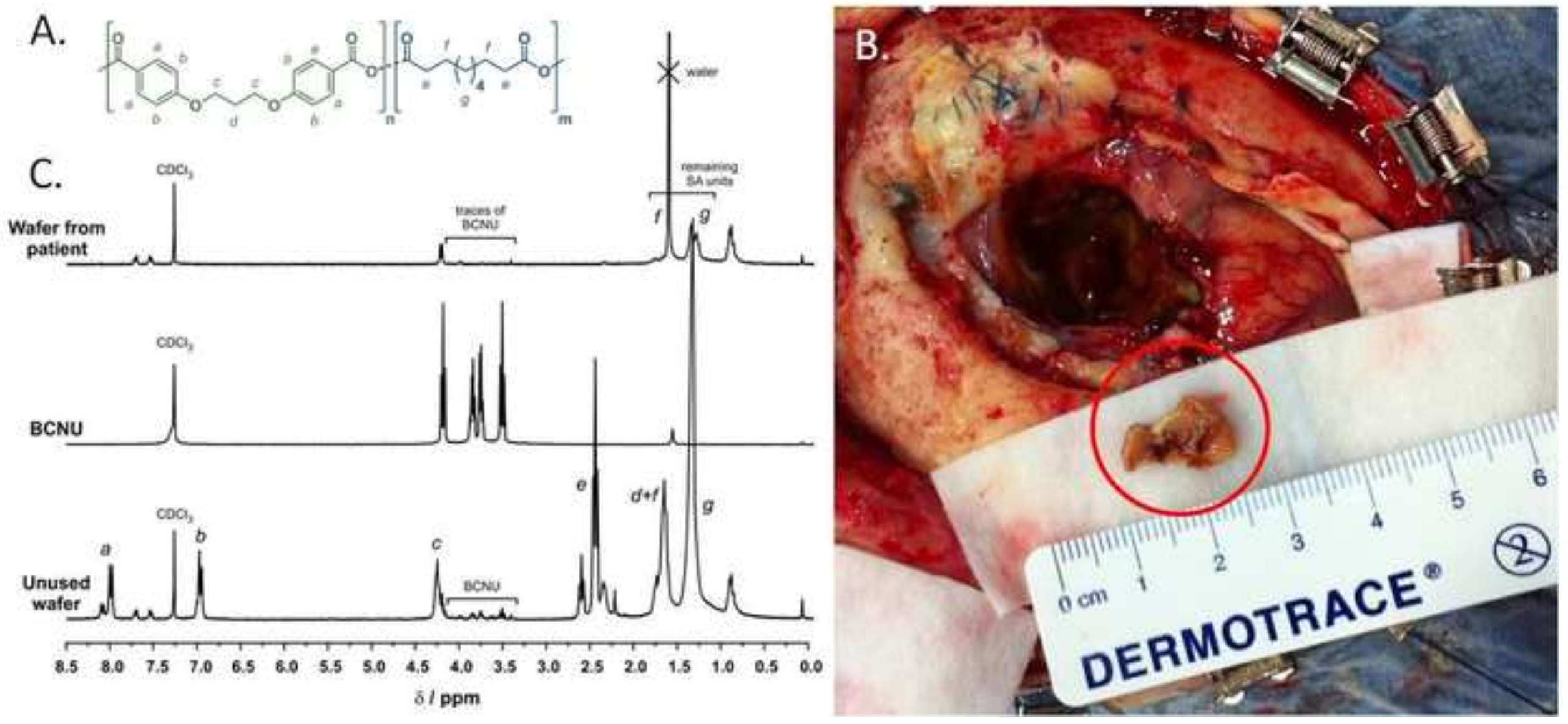


\section{Carmustine wafer}

\section{Unexpected non-degraded copolymer}

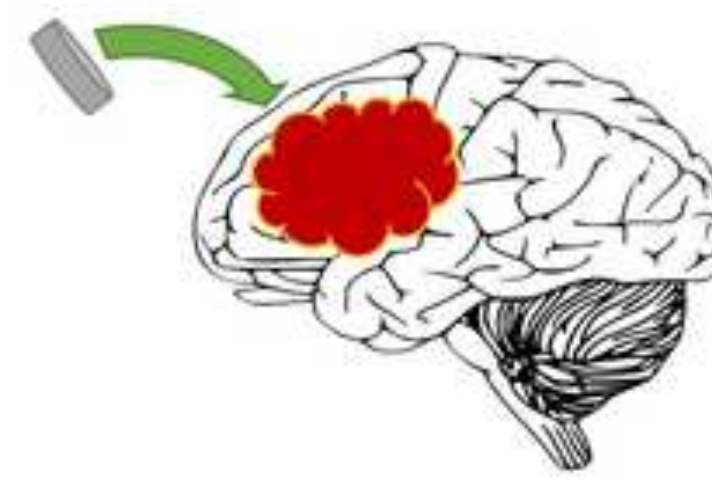

11 months

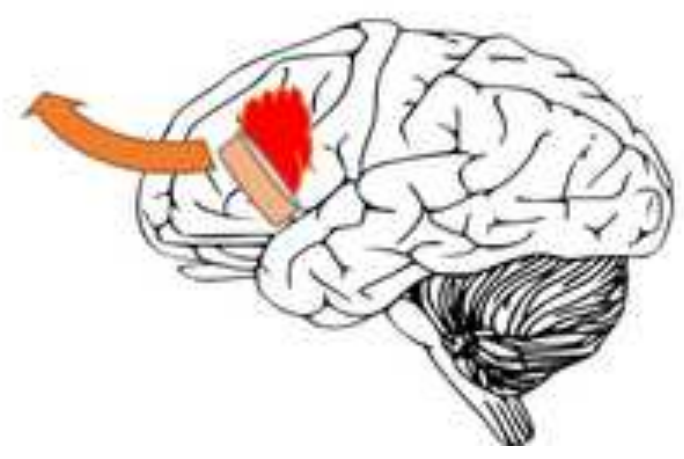

